\title{
Neuromuscular Maturity Assessment
}

National Cancer Institute

\section{Source}

National Cancer Institute. Neuromuscular Maturity Assessment. NCI Thesaurus. Code C89516.

A neuromuscular examination of a newborn's developmental characteristics. 